\title{
Nota nomenclatural sobre Amastris stali Fowler, nom. nud. (Hemiptera, Auchenorrhyncha, Membracidae)
}

\section{Gabriel Simões de Andrade ${ }^{1}$}

\begin{abstract}
Nomenclatural note about Amastris stali fowler, nom. nud. (Hemiptera, Auchenorrhyncha, Membracidae). Amastris stali Fowler, 1895, nom. nud. is here considered.

KEY WORDS. Hemiptera, Auchenorrhyncha, Membracidae, Amastris
\end{abstract}

Amastris stali Fowler, 1895, nom. nud.

Amastris stali Fowler, 1895: 94.

Amastris stali; Metcalf \& Wade, 1965: 860 (cat.; =C. cavicornis Stål, 1859); error.

FOWLER (1895) fez referência a este nome como o de uma espécie que seria descrita por F.W. Goding. O exame dos trabalhos deste autor e da revisão do gênero Amastris Stål, 1862, feita por BRoOMFIELD (1976), mostrou que a espécie associada a este nome não foi publicada.

O estudo da citação deste nome no catálogo de METCALF \& WADE (1965) mostrou que ela foi considerada erroneamente como uma nova combinação para Ceresa stalii Butler, 1877 no gênero Amastris e não uma interpretação correta do binômio publicado por FowLER (1895).

\section{REFERÊNCIAS BIBLIOGRÁFICAS}

BRoOMFIELD, P.S. 1976. A revision of the genus Amastris (Homoptera: Membracidae). Bull. Br. Mus. nat. Hist. (Ent.) 33 (4): 347-460.

FOWLER, W.W. 1895. Order Rhynchota. Suborder Hemiptera-Homoptera. Biologia Cent.-Amer. 2: 89-112.

MetCalf, Z.P. \& V. WADE. 1965. General catalogue of the Homoptera. A supplement to Fascicle I - Membracidae of the General catalogue of Hemiptera. Membracoidea. In two sections. Raleigh, North Carolina State University, 1552p.

Recebido em 31.X.1997; aceito em 01.III.1999

1) Departamento de Ciências Biológicas, Universidade Estadual do Oeste do Paraná. Rua Universitária 2069, Caixa Postal 711, 85814-110 Cascavel, Paraná, Brasil. 